Volume 8. No. 9, September 2020

International Journal of Emerging Trends in Engineering Research

Available Online at http://www.warse.org/IJETER/static/pdf/file/ijeter102892020.pdf

https://doi.org/10.30534/ijeter/2020/102892020

\title{
Breast Cancer Diagnosis Using MLP Back Propagation
}

\author{
Pachipala Yellamma $^{1}$, Ch Smitha Chowdary ${ }^{2}$, G Karunakar ${ }^{3}$, B.V Subba Rao ${ }^{4}$, Vithya Ganesan ${ }^{5}$ \\ 1,2 Associate Professor, Department of Computer Science and Engineering, Koneru Lakshmaiah Education \\ Foundation, Vaddeswaram, AP, India, pachipala.yamuna@gmail.com \\ ${ }^{3}$ Assistant Professor, Department of Computer Science and Engineering, Koneru Lakshmaiah Education \\ Foundation, Vaddeswaram, AP, India \\ ${ }^{4}$ Professor, Department of Information Technology, P.V.P Siddhartha Institute of Technology, Vijayawada, A.P, \\ India, \\ ${ }^{5}$ Professor, Department of Computer Science and Engineering, Koneru Lakshmaiah Education Foundation, \\ Vaddeswaram, AP, India \\ Corresponding author: pachipala.yamuna@gmail.com
}

\begin{abstract}
Breast Cancer (BC) is one of the most prevalent forms of Cancer among women. Premature diagnosis of BC is crucial to the survival of the patient. Here we implement an algorithm designed to diagnose and forecast breast cancer using a multi-layer perceptron (MLP) back-propagation technique that will help doctors diagnose the disease (benign, malignant). The proposed MLP includes an input layer, and, has inputs linked to the ten attributes of the data set. It has a hidden layer with five nodes (neurons). It leads to the pair outcomes: benign and malignant. The objective of our projected algorithm is to diagnose and classify the disease. MLP can help timely recognition of the cancer, and, therefore, can help to go for proper medication at early stage of cancerous development. This approach is tested on the (WBC) Wisconsin Breast Cancer dataset, resulted in 98.9 percent accuracy of classification using MLP back propagation.
\end{abstract}

Key words: Neural-Network, Tumour, Prediction, Features, Training, Analysis, Multi-layer Perceptron

\section{INTRODUCTION}

As per the reports, one of each eight women in the United States build ups breast cancer in their life span. It is one of the most critical diseases among women leading to their death. Premature identification needs an exact and consistent diagnosis system that allows physician to differentiate benign breast tumours from malignant ones without departing for surgical biopsy. Marcano-Cedeno et al. (2011) Breast cancer originates with an unrestrained partition of one cell and consequences in an observable mass called tumour [1]. The tumour can be benign or malignant. Katsis et at (2013) Breast Cancer causes several of the risk factors such as genetic, obesity, family history, having a first child after the age thirty, not having children, aging, menstrual periods, not having children, smoking, drinking, that raise a women possibility of developing breast cancer [2]. Anil Arora et al. (2016) the precise diagnosis of the breast cancer is one of the critical problems in the medical field [3]. NN based MLP back propagation technique seems to be an efficient method for classification of breast cancer. This approach is pedestal on the WBC (Wisconsin Breast Cancer) and the taxonomy of dissimilar category of breast cancer datasets. The MLP back propagation is used to reduce the error rate of breast cancer and increase accuracy.

\section{MATERIALS AND METHOD}

Related work discloses that different methods employed for diagnosis of Breast Cancer by using NN approaches particularly in medical applications. It is intended for assessment; this procedure is extremely complicated to obtain a result as of which schema is better one as it depends on numerous factors similar to number of occurrences to be inspected, kind of abnormalities and dataset being used.

Alaa Rateb Mahmoud Al-shamasneh et al. 2017; Ali Abd Elgader et al. 2011 Described the various NN model for categorize WBC datasets are MLP, Generalized Regression Neural Network (GRNN) and Probabilistic Neural Networks (PNN) [4][5]. The overall results examining that MLP and GRNN are best appropriate for NN model for classifying WBC dataset. Azar et al. (2011) Used the MLP an obtained an accuracy of 96.18 percent [6]. Basavaraj hiremath et al. 
Pachipala Yellamma et al., International Journal of Emerging Trends in Engineering Research, 8(9), September 2020, 5539 - 5544

(2015) Proposed an Automated Evaluation of Breast Cancer Detection on image processing morphological algorithm produced an accuracy of 88.33 percent [7]. Bichen et al. (2014) proposed a new K-SVM (K-Means and Support Vector Machine) algorithm was performed the accuracy of 97.3 percent, when tested on the WBC [8]. Chandra Prasetyo Utomo et al. (2014) a result showed that Extreme Learning Machine Neural Networks has better performances in term of sensitivity and accuracy in all experiments and with accuracy of more than 95percent[9][10]. Hui-Ling Chen et al. (2011) projected a rough set based supporting vector machine classifier [11]. The Rough set reduction algorithm used for features selection followed by support vector machine in order to achieve the highest classification accuracy 99.4 percent, 100 percent, and 100 percent for partition of training and test (50:50) percent of training and test partition (70:30), and percent of training and test partitions (80:20) respectively, for a subset that restricted five features. Janghel et al. (2010) used MLP with Gradient Descent (GD) function to get a low accuracy of 51.9 percent [12]. Jung et al. (2015) used MLP with back propagation training algorithm on ultrasound images, experimental studies obtained high accuracy for detection and classification of breast cancer on 96 percent, 95 percent [13]. Kasra et al. (2015) used a new algorithm based on $\mathrm{C} 4.5$ on $\mathrm{WBC}$ which is based on entropy of the attribute and a comparison made with weka tool and results having an accuracy of 97.9 percent [14][15]. Raad, et al, (2012) employed the MLP back propagation function achieved an accuracy of 88 percent [16]. Sarvestan Soltani et al. 2010; Smaranda Belciug 2010 Discussed different NN techniques such as Probabilistic Neural Network, Radial Basis Function (RBF) Self Organizing Map and MLP are used to classify the WBC dataset and then compared all the networks according to their accuracy[17]. According to his results RBF and PNN are shown as the good classifiers in the training set [18][19].Seema Singh et al. (2014) employed MLP algorithm obtained accuracy of more than 95 percent [20]. Usha et al. (2010) the investigational outcomes were based on single and multi layer NN models [21][22]. BP algorithm and MLP was adopted to produce an accuracy of 92 percent. Y. Wu et al. (2006) explain the Multilayer Perceptron architecture based on parameter regularization and cross-validation [23][24]. This gets the least absolute error and qualified error ratio. The main cause of breast cancer is when a single cell or group of cells escapes from the usual controls, that regulate cellular growth and begins to multiply and spread. Some tumours are malignant that means the abnormal growth invades the surrounding tissues and that may metastasize or spread to remote areas of the body. The benign masses may lead to complications whereas malignant tumors are serious cancer. The dataset used in this paper is publicly available and was created by Dr. William H. Wolberg, physician at the University of Wisconsin Hospital at Madison, Wisconsin, USA[25][26]. Health records may include a range of data such as general medical records, patient examinations, patient treatments, medical history, allergies, immunization status, laboratory results, radiology images, and some useful information for examination. This rich information may help researchers in examining and diagnosing diseases using computer techniques. Features are computed from a digitized image of a fine needle aspirate (FNA) of a breast mass. They describe characteristics of the cell nuclei present in the image. Number of instances: 569, Number of attributes: 32 (ID, diagnosis, 30 real-valued input features). This has 699 instances (Benign: 458 Malignant: 241) of which 16 instances has missing attribute values removing that we have 683 instances of which 444 benign and 239 are malignant. Features are computed from a digitized image of a Fine Needle Aspiration (FNA) of a breast mass. Table 1 and Table 2 show the dataset description and the sample dataset representation for Breast Cancer stage and Table 3 shows the sample dataset for experimentation respectively.

Table 1: Breast Cancer dataset attribute description

\begin{tabular}{|c|c|c|}
\hline $\begin{array}{c}\text { S.N } \\
\mathbf{O}\end{array}$ & $\begin{array}{l}\text { Attribute } \\
\text { name }\end{array}$ & Attribute description \\
\hline 1 & $\begin{array}{l}\text { Clump } \\
\text { Thickness }\end{array}$ & $\begin{array}{l}\text { Malignant cells are multi layered while } \\
\text { benign cells are layered in a single band }\end{array}$ \\
\hline 2 & $\begin{array}{l}\text { Uniformity of } \\
\text { cell size }\end{array}$ & $\begin{array}{l}\text { Cancer cells tend to vary in size. That is } \\
\text { why this feature is valuable in saying } \\
\text { whether the cells are cancerous or not }\end{array}$ \\
\hline 3 & $\begin{array}{l}\text { Uniformity of } \\
\text { cell shape }\end{array}$ & $\begin{array}{l}\text { Cancer cells tend to vary in shape. That } \\
\text { is why this feature helps to know if the } \\
\text { cell is cancerous or not. }\end{array}$ \\
\hline 4 & $\begin{array}{l}\text { Marginal } \\
\text { Adhesion }\end{array}$ & $\begin{array}{l}\text { Normal blood cells adhere to each } \\
\text { other. In cancer cell this ability } \\
\text { diminishes. So loss of adhesion is a sign } \\
\text { of malignancy }\end{array}$ \\
\hline 5 & $\begin{array}{c}\text { Single } \\
\text { epithelial cell } \\
\text { size }\end{array}$ & $\begin{array}{c}\text { Epithelial cells that are enlarged } \\
\text { significantly t out to be } \\
\text { malignant }\end{array}$ \\
\hline 6 & Bare nuclei & $\begin{array}{l}\text { Term for nuclei not surrounded by } \\
\text { cytoplasm. They are typically seen in } \\
\text { harmless tumors }\end{array}$ \\
\hline 7 & $\begin{array}{c}\text { Bland } \\
\text { Chromatin }\end{array}$ & $\begin{array}{l}\text { In malignant cells the chromatin is } \\
\text { found to be rough in texture, while the } \\
\text { nucleuses of noncancerous cells have } \\
\text { an unvarying texture. }\end{array}$ \\
\hline 8 & $\begin{array}{l}\text { Normal } \\
\text { Nucleoli }\end{array}$ & $\begin{array}{l}\text { In malignant cells the nucleoli are } \\
\text { largely visible, and are sometimes }\end{array}$ \\
\hline
\end{tabular}


Pachipala Yellamma et al., International Journal of Emerging Trends in Engineering Research, 8(9), September 2020, 5539 - 5544

\begin{tabular}{|c|l|l|}
\hline & & $\begin{array}{l}\text { present in a greater quantity. In } \\
\text { contrast, benign cells have Microscopic } \\
\text { nucleoli. }\end{array}$ \\
\hline 9 & Mitosis & $\begin{array}{l}\text { Mitosis is a process that is inherently } \\
\text { managed by the genes inside every cell. } \\
\text { If this control fails, a single cell can } \\
\text { multiply to make new Cells that can } \\
\text { lose control and are cancerous. }\end{array}$ \\
\hline
\end{tabular}

Table 2: Dataset Attribute description

\begin{tabular}{|c|c|c|}
\hline $\begin{array}{c}\text { S.N } \\
\text { O }\end{array}$ & Attribute Description & Representation \\
\hline 1 & Clump Thickness: $1-10$ & A \\
\hline 2 & Uniformity of Cell Size: $1-10$ & B \\
\hline 3 & Uniformity of Cell Shape: $1-10$ & C \\
\hline 4 & Marginal Adhesion: $1-10$ & D \\
\hline 5 & Single Epithelial Cell Size: $1-$ & E \\
\hline 6 & 10 & F \\
\hline 7 & Bare Nuclei: $1-10$ & G \\
\hline 8 & Normal Nucleoli: $1-10$ & H \\
\hline 9 & Mitoses: $1-10$ & I \\
\hline 10 & Class benign, malignant \\
\hline
\end{tabular}

Table 3: Sample dataset

\begin{tabular}{|c|c|c|c|c|c|c|c|c|c|}
\hline A & B & C & D & E & F & G & H & I & CLASS \\
\hline 7 & 3 & 2 & 10 & 5 & 10 & 5 & 4 & 4 & Malignant \\
\hline 6 & 1 & 1 & 1 & 2 & 1 & 3 & 1 & 1 & Benign \\
\hline 10 & 7 & 7 & 6 & 4 & 10 & 4 & 1 & 2 & Malignant \\
\hline 4 & 1 & 1 & 1 & 2 & 1 & 2 & 1 & 1 & Benign \\
\hline 4 & 1 & 1 & 1 & 2 & 1 & 2 & 1 & 1 & Benign \\
\hline 7 & 4 & 6 & 4 & 6 & 1 & 4 & 3 & 1 & Malignant \\
\hline 8 & 7 & 6 & 4 & 5 & 1 & 4 & 2 & 1 & Malignant \\
\hline 1 & 1 & 1 & 1 & 2 & 2 & 1 & 3 & 2 & Benign \\
\hline 9 & 5 & 6 & 10 & 4 & 2 & 4 & 1 & 2 & Malignant \\
\hline 2 & 1 & 1 & 1 & 2 & 1 & 2 & 1 & 2 & Benign \\
\hline 4 & 2 & 1 & 2 & 1 & 2 & 2 & 3 & 1 & Benign \\
\hline 1 & 1 & 1 & 1 & 2 & 9 & 1 & 2 & 1 & Benign \\
\hline 8 & 9 & 10 & 5 & 6 & 4 & 4 & 1 & 3 & Malignant \\
\hline 5 & 4 & 4 & 2 & 3 & 4 & 3 & 2 & 3 & Benign \\
\hline 4 & 3 & 2 & 2 & 1 & 2 & 3 & 1 & 2 & Benign \\
\hline 4 & 3 & 2 & 4 & 2 & 5 & 6 & 2 & 3 & Malignant \\
\hline 1 & 2 & 3 & 4 & 5 & 4 & 5 & 1 & 2 & Malignant \\
\hline 1 & 2 & 3 & 8 & 9 & 4 & 1 & 2 & 2 & Benign \\
\hline 4 & 5 & 2 & 3 & 1 & 2 & 2 & 3 & 4 & Benign \\
\hline 1 & 2 & 3 & 4 & 5 & 1 & 2 & 2 & 1 & Benign \\
\hline
\end{tabular}

In this paper MLP back propagation algorithm having three layers such input layer, a hidden layer and an output layer. The proposed network processing time can be compact on each layer in the network; as a result the overall training period reduces. The proposed breast cancer schema prediction and diagnosis is depends on ten features, normalization, leaning rate and activation function is applied throughout the training process.

This work proposes a technique to predict the existence of Breast-Cancer with women using a Neural Network (NN) technique with efficient algorithm using 10 attributes as an input of WBC dataset consisting of samples of 699 patients. The main reason for using back propagation algorithm is to achieve high accuracy of prediction, and, thereby, minimizing the error rate. The proposed algorithm tries to minimize the error on a given weights space by the means of GD. The objective of our proposed MLP is to make a model that exactly applies the input data to the output data by using a past dataset. Because of that, the model can be used to generate the output when the desired output is unidentified. In Figure 1 shown the proposed MLP neural network model has been represented in Figure 1. The algorithm corresponding Breast cancer disease prediction has been given by Algorithm 1 .

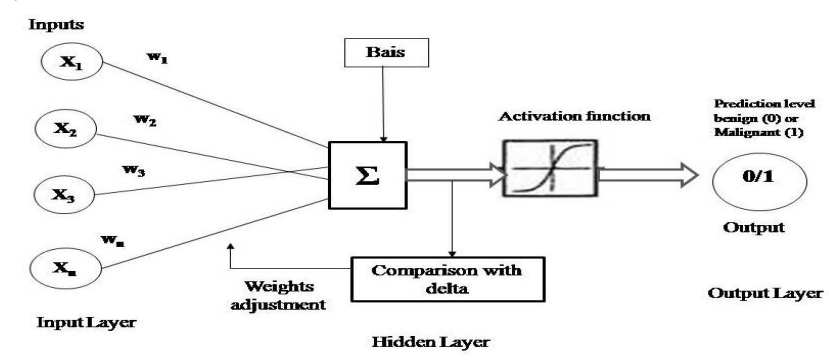

Figure 1: Multi Layer Perceptron NN model Breast cancer Disease Prediction: Analysis flow Datasets for Breast Cancer has been taken from the WBC neural network repository as the concerned data set has already been pre-processed. The concerned algorithm makes use of the ten features shown in the Table 4.

Table 4: Features information

\begin{tabular}{|c|c|c|}
\hline$\#$ & Features & Domain \\
\hline 1 & Sample code number & 0.1 to 1 \\
\hline 2 & Bare Nuclei & 0.1 to 1 \\
\hline 3 & Bland Chromatin & 0.1 to 1 \\
\hline 4 & clump thickness & 0.1 to 1 \\
\hline 5 & Marginal Adhesion & 0.1 to 1 \\
\hline 6 & Single Epithelial Cell Size & 0.1 to 1 \\
\hline 7 & Uniformity of Cell Size & 0.1 to 1 \\
\hline 8 & Uniformity of Cell Shape & 0.1 to 1 \\
\hline 9 & Normal nucleoli & 0.1 to 1 \\
\hline 10 & mitosis & 0.1 to 1 \\
\hline
\end{tabular}

Next, data clean up procedure is applied and the outcome is utilized as key to the NNBP (Neural-Network-Back-Propagation) algorithm for predicting the cost function (sigmoid function). In Figure 2 
Pachipala Yellamma et al., International Journal of Emerging Trends in Engineering Research, 8(9), September 2020, 5539 - 5544

shown the breast cancer prediction analysis flow is exposed clearly. The output is shown after predetermined iterations.

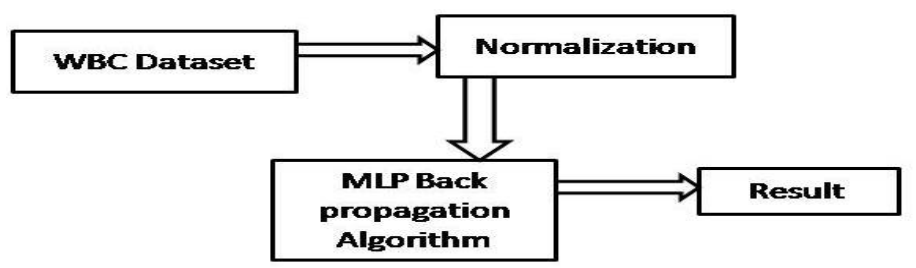

Figure 2: Breast cancer Disease Prediction analysis flow

\section{Data Normalization}

In neural networks the most significant pre-processing step is data normalization. In order to get better performance of a MLP neural network, we normalize the data in the first phase. After the processing the data set using given algorithm, we get output in the interval [0 1].An imaginative appearance of the input data was offered in proposed study was in the analog form, the range starting from 0.1 to 1 . Translating the input data into digital form, and employs it as input to NN. Scaling or normalization must be recognized for each and every feature. The ten features in the analog appearance are scale in the range of 0 to 1 .New value (After normalization) is equal to current value minus minimum value divided by maximum minus minimum value. Breast cancer prediction is analyzed by using MLP back propagation algorithm.

\section{Algorithm 1: breast cancer disease prediction}

Function model $=$ ExtentedMLP $($ InputAttribute, Target, rate, NoIter, visualize)

Input: All 10 attributes

Output: learned model [(Benign (non-cancerous) or

Malignant (cancerous))]

\section{Step 1: Initialization}

$[\mathrm{p}, \mathrm{q}]=$ size (InputAttribute);

Bais $=$ ones $(\mathrm{p}, 1)$;

No_Of_Input_Nodes $=\mathrm{q}+1$;

No_Of_HiddenLayer_Nodes= No_Of_Input_Nodes;

\section{Step2: Generate Random Weights}

Weights_Input_Hidden $=$ (randn (No_Of_Input_Nodes,

No_Of_HiddenLayer_Nodes) - 0.5)/10;

Weights_Hidden_Output $=(\operatorname{randn}(1$,

No_Of_HiddenLayer_Nodes) - 0.5)/10;

\section{Step 3: Execution}

Hidden layer size $=1$;

For iter $=1$ : NoIter

rand_Input $=$ rand_perm $(\mathrm{p}, \mathrm{p})$;

For $\mathrm{i}=1$ : $\mathrm{p}$;

\section{Step4: Setting perceptron Parameters}

Input = InputAttribute (rand_Input (i), :);

\author{
Target $=$ Target_vector $($ rand_Input $(\mathrm{i}), 1)$; \\ HiddenInput $=\tanh ($ Input $*$ Weights_Input_Hidden $)$; \\ HiddenOutput $=$ HiddenInput $*$ \\ Weights_Hidden_Output' \\ Error $=$ HiddenOutput - Target; \\ Options $=$ maxIter $=10$ \\ Delta $=0.001$
}

\section{Step 5: calculate error function}

Delta $=$ error $*$ rate $*$ HiddenInput;

Weights_Hidden_Output $=$ Weights_Hidden_Output delta;

Delta $=($ rate/10) $*$ error * Weights_Hidden_Output $' *$

(1-(HiddenInput'^2)) * Input;

Weights_Input_Hidden $=$ Weights_Input_Hidden -

delta'; End

Step 6: Predict Level of breast cancer disease

Pred $=$ Weights_Hidden_Output $*$ tanh (InputAttribute *

Weights_Input_Hidden)';

Err $($ iter $)=\left(\text { sum }\left((\text { pred' }- \text { Target })^{\wedge} 2\right)\right)^{\wedge} 0.5$;

If err (iter) $<0.001$ end

Step 7: Display

Training Set Accuracy, error

\section{RESULTS AND DISCUSSION}

Our scheme categorizes a tumour as benign or malignant based on WBC dataset, it contain samples of 699 patients having 10 features as has been given in the table 1 , and, one class feature. The class allocation is 65.5 percent (458 samples) for benign and 34.5 percent (241 samples) for malignant.

\section{Training and Classification}

Our proposed Multi Layer perceptron uses an algorithm called back-propagation for training. Breast cancer is forecasted using the NN technique using the proposed algorithm using 10 parameters as input from WBC data set (consisting of samples of 699 patients). Here, the input data is repetitively offered to the NN. With each appearance a hidden layer result of the neural network compared with the target result and then, error is calculated. The resultant error is back propagated to the $\mathrm{NN}$ and then, used to normalize the weights, so that the error gets reduced after the end of each of the iterations. This training technique is capable to solve nonlinearly separable problems. The solution to the learning problem is considered as a combination of various weights leading to the minimization of the error rate function. As the gradient method needs to perform a number of procedures for the error function at each and every execution step, there is a 
Pachipala Yellamma et al., International Journal of Emerging Trends in Engineering Research, 8(9), September 2020, 5539 - 5544

need to guarantee differentiability for error function and its continuance in each of the iteration. The key reason intended for using back-propagation algorithm is to reach high accuracy of prediction by minimizing the error rate. The algorithm tries to minimize the error function in a given weight space by means of the gradient descent.

\section{Performance of MLP Back Propagation Algorithm}

Analysis, construction and learning, three various phases are used in categorization of the disease. Three layers are present in the network of back propagation, input layer consisting of ten features, hidden layer possessing five neurons and output layer having a single class (benign or malignant). The significance of neurons of the output layer specifies, if the output corresponds to a Cancer case or not. Weights of the network connections are positioned arbitrarily in learning stage. The input layer attributes standardized values between zero (0) and one (1). Table 5 represents classification result of Breast Cancer using MLP.

Table 5: Experimental result on Breast Cancer using Multilayer Perceptron Back propagation

\begin{tabular}{|c|c|c|}
\hline $\begin{array}{c}\text { TRAINING SET } \\
\text { SAMPLES }\end{array}$ & $\begin{array}{c}\text { TEST SET } \\
\text { SAMPLES }\end{array}$ & $\begin{array}{c}\text { CLASSIFICATION } \\
\text { EFFICIENCY OF MLP }\end{array}$ \\
\hline 378 & 120 & $98.9 \%$ \\
\hline 180 & 100 & $95.1 \%$ \\
\hline 150 & 178 & $92.0 \%$ \\
\hline 120 & 378 & $89.4 \%$ \\
\hline
\end{tabular}

Here eighty nine percent of patients have been classified properly, in this case, in which the training set samples (120) are less than the test set samples (378). In this case the error is twelve percent. Input to the Breast cancer diagnosis is a 10x699 matrix describing 10 features of the samples consisting of the 699 patients. The breast cancer goal is a 2x699 matrix where every column specifies an accurate class attribute with a one in either benign or malignant. Among these circumstances, the input and target matrix will be arbitrarily divided into two datasets.

Table 6: Description of WBC database

\begin{tabular}{|c|c|c|}
\hline S.No & Features & Number of attributes \\
\hline 1 & Number of samples & 699 \\
\hline 2 & Number of features & 10 \\
\hline 3 & $\begin{array}{c}\text { Attributes 2 through } \\
10\end{array}$ & Samples \\
\hline 4 & Classes & $\begin{array}{c}\text { 1.benign with value 0 } \\
\text { 2.malignant with value 1 }\end{array}$ \\
\hline 5 & Class Distribution & $\begin{array}{c}\text { Benign is 458 samples (65.5\%) } \\
\text { Malignant is 241 samples } \\
(34.5 \%)\end{array}$ \\
\hline
\end{tabular}

Description of WBC data attributes has been represented in the Table 6 . The input and target matrix will be arbitrarily divided into two datasets. Those are training and testing set. Eighty percent of samples are used for the training and remaining twenty percent of samples are used for the testing. In the beginning of the training, all the weights are place randomly in NN. The hidden layer assigns 5 neurons. The output layer gives two outputs. The training carries on for 10 iterations. All the computations have been executed by using MATLAB 9.0 neural network toolbox.

\section{IV.CONCLUSION}

In this investigation, the performance of MLP has been studied on a real life women breast cancer problem. The investigational outcomes in Wisconsin breast cancer dataset show that MLP back propagation is an efficient classification algorithm for training and testing. MLP gives the accurate classification and analysis when the dataset is measured. This model has specified a good breast cancer diagnosis performance of 98.9 percent.

\section{V.FUTURE SCOPE}

In real life, medical inspections and reports will take a lot of time consuming process scheduled by specialized doctors. In this report, time spending procedure is made by in a few minutes and details will generate in a short span of time afterwards patient's treatment will make further and quicker.

\section{REFERENCES}

1. A.Marcano-Cedeno ,J. Quintanilla-Domínguez, D. Andina. WBCD breast cancer database classification applying artificial metaplasticity, neural network' Expert Systems with Applications.,vol. 38, 9573-9579.2011.

2. C.D. Katsis, I. Gkogkou, C.A. Papadopoulos, P.V. Boufounou, G. Stylios, Y. Goletsis, Using Artificial Immune Recognition Systems in Order to Detect Early Breast Cancer, I.J. Intelligent Systems and Applications, 02, 34-40.2013.

3. Anil Arora, Kalpana Kaushik ., Breast Cancer Diagnosis using Artificial Neural Network, International Journal of Latest Trends in Engineering and Technology (IJLTET) ISSN: 2278-621X, Vol 7 issue 2 July. 2016.

4. Alaa Rateb Mahmoud Al-shamasneh, Unaizah Hanum Binti Obaidellah. Artificial Intelligence Techniques for Cancer Detection and Classification: Review Study, European Scientific Journal., vol.13, No.3 ISSN: 1857 - 7881 (Print) e - ISSN 1857- 7431.2017.

5. Pachipala Yellamma, Dileep Kumar, P.Sai Pradeep Reddy, K.Sri Harsha, L.Jagadeesh, N. Probability of data leakage in cloud computing, International Journal of Advanced Science and Technology ., Vol.29, No. 6, (2020), pp. 3444-3450.2020 
Pachipala Yellamma et al., International Journal of Emerging Trends in Engineering Research, 8(9), September 2020, 5539 - 5544

6. Azar, A. T., \& El-Said, S. A. Probabilistic neural network for breast cancer classification. Neural Computing and Applications, 23(6), 1737-1751.2013.

7. Basavaraj Hiremath and SC Prasannakumar, Automated Evaluation of Breast Cancer Detection Using SVM Classifier., IJCSEITR, ISSN(P): 2249-6831; ISSN(E): 2249-7943, Vol. 5, Issue 1, Feb, 11-20 17.

8. Bichen Zheng, Sang Won Yoon, Sarah S. Lam ,Breast cancer diagnosis based on feature Extraction using a hybrid of K-means and support vector machine algorithms., Expert Systems with Applications 41 ,1476-1482,ELSEVIER,2014

9. Chandra Prasetyo Utomo, Aan Kardiana, Rika Yuliwulandar , Breast Cancer Diagnosis using Artificial Neural Networks with Extreme Learning Technique. International Journal of Advanced Research in Artificial Intelligence (IJARAI) ,Vol. 3, No. 7, 2014.

10. Pachipala Yellamma, P.S. S. Rajesh, V.V.S.M.Pradeep, Y.B.Manishankar., Privacy Preserving Biometric Authentication and Identification in Cloud Computing., International Journal of Advanced Science and Technology .,Vol. 29, No. 6, (2020), pp. 30873096,2020.

11. Hui-Ling Chen,Bo Yang Jie Liu Da-You Liu, A support vector machine classifier with rough set-based feature selection for breast cancer diagnosis,. Expert Systems with Applications, vol. 38,9014-9022, ,ELSEVIER,2011.

12. Janghel, R., Shukla, A., Tiwari, R., \& Kala, R. Breast cancer diagnosis using artificial neural network models. The Information Sciences and Interaction Sciences (ICIS), $20103^{\text {rd }}$ International Conference on. 2010

13. Jung, I.-S. Thapa,D., \& Wang, G.-N. Neural Network Based Algorithms for Diagnosis and Classification of Breast Cancer Tumor. Computational Intelligence and Security-Springer Berlin Heidelberg., Vol.3801, pp. 107-114,.2015.

14. Kasra Madadipouya, A New Decision Tree Method For Data Mining In Medicine, Advanced Computational Intelligence: An International Journal (ACII), Vol.2, No.3, July.,2015

15. Pachipala Yellamma, Challa Narasimham. Data Security In Cloud Using Rsa, 2013 IEEE International Conference on Computing, Communication and Networking Technologies, published in IEEE Xplore Digital library ,ISBN 978-1-4799-3925-1

16. Raad, A., Kalakech, A., \& Ayache, M. Breast cancer classification using neural network approach: MLP and RBF. Networks, 7(8), 9.2012

17. Sarvestan Soltani A, Safavi A A, Parandeh M N and Salehi M . Predicting Breast Cancer Survivability using Data Mining Techniques, Software Technology and Engineering (ICSTE), 2ndInternational Conference, Vol.2, pages 227-231.2010.

18. Smaranda Belciug, A two stage decision model for breast cancer detection, Annals of the University of
Craiova, Mathematics and Computer Science Series Volume 37(2), Pages 27-37 ISSN: 1223-6934,2010.

19. Seema Singh, S. H., Harini J and Surabhi B.R. An Efficient Neural Network Based System for Diagnosis of Breast Cancer,. International Journal of Computer Science and information Technologies,5 (((3)), 4354-4360.2014

20. Pachipala Yellamma, P.Anupama, K.Lakshmibhavani, U.Jhansi Siva Priya, Ch, Kazalalitha, Implementation Of E-Voting System Using Block Chain Technology, Journal of Critical Reviews., ISSN- 2394-5125 Vol 7, Issue 6, 2020

21. Usha Rani ,.Parallel Approach for Diagnosis of Breast Cancer using Neural Network Technique" International Journal of Computer Applications .(0975 8887), Volume 10- No.3, November-2010.

22. Meghana $T$,Sunanda Nalajala, Manoj Kumar, Jagadeesh, Privacy Preserving Using Pup-Rup Model, International Conference on Intelligent Sustainable Systems(ICISS 2019), IEEE 2019.

23. Yunfeng Wu, Cong Wang, S.C. Ng, Anant Madabhushi, and Yixin Zhong (2006) Breast Cancer Diagnosis Using Neural-Based Linear Fusion Strategies' ICONIP 2006, Part III, LNCS,. 4234, pp. 165 - 175. Springer-Verlag Berlin Heidelberg .

24. Pachipala Yellamma, C. Madhav Bharadwaj, Pakalapati Narendra,G. Leela Sree, and K. Praveen Reddy. Interactive Video Gaming with Internet of Things, Proceeding of the International Conference on Computer Networks, Big Data and IoT (ICCBI - 2018), , Springer Nature Switzerland AG 2020,A. P. Pandian et al. (Eds.): ICCBI 2018, LNDECT 31, pp. 1-10, 2020.

25. Prathyusha Chalasani, S Rajesh, Lung CT Image Recognition using Deep Learning Techniques to Detect Lung Cancer, International Journal of Emerging Trends in Engineering Research (IJETER), VOL.8(8),pp. 3575 - $3579,2020$.

26. Dr. D. Nagajyothi, Rakshith Addagudi, Tejaswini Gunda , Sindhu santhoshi Logitla and G.Arun, Detection of Lung Cancer using SVM Classifier, International Journal of Emerging Trends in Engineering Research (IJETER),VOL.8(5) ,pp. 2177 2180,2020 .

27. Deepa. K, LekhaSree. R. K, Renuga Devi. B, Sadhana. V and Virgin Jenifer. S, Cervical Cancer Classification, International Journal of Emerging Trends in Engineering Research (IJETER),VOL.8(3) ,pp. 804 807,2020 . 